\title{
Discutindo o modelo de Ptolomeu e sua equivalência com o modelo de Copérnico
}

\author{
Discussing Ptolemy's model and its equivalence with the Copernican model
}

\author{
Alan Miguel Velásquez-Toribio*1], Marcos Oliveira ${ }^{2}$ \\ ${ }^{1}$ Universidade Federal do Espirito Santo, Centro de Astrofísica e Cosmologia, Vitória, ES, Brasil \\ ${ }^{2}$ Universidade Federal do Espirito Santo, Departamento de Física, Vitória, ES, Brasil
}

\begin{abstract}
Recebido em 17 de Outubro de 2019. Revisado em 23 de Janeiro de 2020. Aceito em 23 de Janeiro de 2020.
Revisamos o modelo geocêntrico de Ptolomeu e mostramos paso a paso como determinar a longitude de um planeta usando uma abordagem matemática moderna para facilitar sua compreensão. Também discutimos as considerações de Ptolomeu para fixar o valor da latitude dos planetas. Uma equivalência geométrica entre os modelos de Ptolomeu e Copérnico é apresentada, destacando as restrições com as quais esta equivalência foi construída. Ademais, é discutido os aspectos associados à transição entre o modelo geocêntrico de Ptolomeu e o modelo heliocêntrico de Copérnico. Consideramos aspectos sobre o uso de epiciclos sobre epiciclos, assim como, a questão da precisão do modelo de Ptolomeu em relação ao modelo de Copérnico. Em geral, neste trabalho apresentamos uma visão quantitativa e qualitativa do modelo de Ptolomeu de forma que o leitor tenha a disposição uma formulação matemática simples para determinar a posição dos planetas. Adicionalmente, entender como a proposta de Copérnico, mesmo sendo equivalente do ponto de vista geométrico ao modelo de Ptolomeu, se revelou um modelo fisicamente mais completo e de uma estética (movimento da Terra em torno do Sol) diferente capaz de unificar conceitos.
\end{abstract}

Palavras-chave: Ptolomeu, epiciclo, excêntrico e Copérnico.

\begin{abstract}
We reviewed Ptolemy's geocentric model and showed step by step how to determine the longitude of a planet using a modern mathematical approach to facilitate its understanding. We also discussed Ptolemy's considerations for fixing the planets' latitude value. A geometric equivalence between the Ptolemy and Copernicus models are presented, highlighting the restrictions with which this equivalence was built. Furthermore, the aspects associated with the transition between Ptolemy's geocentric model and Copernicus's heliocentric model are discussed. We considered aspects about the use of epicycles over epicycles, as well as the question of the precision of the Ptolemy model in relation to the Copernicus model. In general, in this work we present a quantitative and qualitative view of Ptolemy's model so that the reader has a simple mathematical formulation available to determine the position of the planets. Additionally, to understand how Copernicus' proposal, even though it is geometrically equivalent to Ptolemy's model, turned out to be a physically more complete model and of a different aesthetic (movement of the Earth around the Sun) capable of unifying concepts.
\end{abstract}

Keywords: Ptolemy, Copernicus, epicycle, eccentric.

\section{Introdução}

Na história da ciência, o modelo do Universo geocêntrico de Ptolomeu (90-168 d.C.) teve uma importância fundamental. Por séculos foi o modelo padrão utilizado para determinar os movimentos dos objetos do cosmos. A referência fundamental deste modelo é o livro publicado por Ptolomeu, conhecido em grego como "Mathematike Syntaxis", em árabe chamado de "Al-Majisti", e nas traduções latinas conhecido como Almagestum, nome com o qual é atualmente conhecido [1].

$\mathrm{Na}$ antiga Grécia, o primeiro modelo matemático da astronomia foi apresentado por Eudoxo, que é atualmente conhecido como o modelo das esferas concêntricas. Este

*Endereço de correspondência: alan.toribio@ufes.br modelo geocêntrico utilizava esferas se movimentando com velocidades constantes en torno do seu centro. Em particular, é notável a construção geométrica proposta para explicar o movimento retrógrado dos planetas. De forma sucinta, observamos que o modelo de Eudoxo tinha como base a combinação de duas esferas se movimentando em sentidos contrários e com os eixos inclinados entre si. O resultado é uma trajetória denominada hipópede. Segundo os comentários de Simplicio (490-560 d.C.) [2] o modelo de Eudoxo resultou como resposta ao célebre desafio proposto por Platão (428-348 a.C.) para salvar os fenômenos. Para detalhes deste modelo revisar a referência [3], onde se discute de forma detalhada a matemática do modelo.

O desafio de salvar os fenômenos implicava em usar unicamente as trajetórias circulares e os movimentos 
com velocidades constantes para explicar as trajetórias dos objetos do cosmos. No entanto, o modelo das esferas concêntricas encontrou rapidamente conflitos com as observações. Por exemplo, todos os objetos no modelo das esferas concêntricas estavam a uma mesma distância da Terra e, assim, a luminosidade dos objetos não poderia mudar. Mas, as observações mostravam que a luminosidade dos objetos do cosmos mudavam ao longo do ano.

Neste contexto, um novo modelo surge para obter uma melhor correspondência com as observações astronômicas, o qual usava como ferramentas geométricas os círculos excêntricos e os epiciclos. A este respeito se destaca o trabalho de Apolônio de Perga (262-194 a.C.) que, aproximadamente no ano 200 a.C., demonstrou um teorema sobre a equivalência entre os movimentos usando círculos excêntricos e epiciclos. Adicionalmente, Apolônio chamou aos pontos onde o planeta mudava de direção de pontos estacionários 4].

Hiparco de Nicea (190-120 a.C.) foi outro astronômo que contribuiu notavelmente. Hiparco é considerado um dos maiores astrônomos da antiguidade. Ele registrou uma grande quantidade de observações, dando origem a um catálogo de estrelas. Ele percebeu, ao comparar dados de diferentes épocas, que as estrelas ao longo dos anos se deslocavam com respeito ao ponto vernal. O ponto vernal corresponde ao ponto pelo qual o Sol passa quando atravessa o equador celeste nos equinócios. Isto permitiu a Hiparco descobrir o fenômeno da precessão dos equinócios. No entanto, é importante destacar que desde o ponto de vista geocêntrico, Hiparco deve ter interpretado a precessão como um movimento do eixo da esfera celeste e não do eixo de rotação da Terra, pois, na concepção antiga, a Terra não se movimentava. Hiparco também foi um grande inventor de instrumentos astronômicos, ele aperfeiçoou o astrolábio, inventou o dioptro, entre outras invenções [4,5].

Por outro lado, Claudio Ptolomeu, no século II d.C, escreveu a obra-mestra da astronomia da antiguidade, o mencionado Almageste 1 . Nesta obra Ptolomeu compilou o conhecimento astronômico dos gregos desenvolvido até o século II d.C. Seu livro Almagesto deve ter sido escrito aproximadamente em 150 d.C. Pouco se conhece sobre a biografia de Ptolomeu. Mas sabemos que no ano 125 d.C., ele estava trabalhando em Alexandria, pois data desta época sua primeira observação astronômica anotada. Na astronomia conhecemos três principais trabalhos de Ptolomeu. O maior trabalho, é sem dúvida, o Almagesto. O outro livro é chamado de Tábuas Práticas 2 onde se apresentam em forma resumida tábuas com parâmetros

${ }^{1}$ Ptolomeu também escreveu a obra-mestra da astrologia da antiguidade, o Tetrabiblios. Esta característica mostra a forte ligação entre a astronomia e a astrologia na época antiga, uma visão completamente diferente da atual.

${ }^{2}$ Tradução livre do livro de Ptolomeu conhecido em inglês como:Handy Tables. Livro posterior ao Almagesto e que na antiguidade e na idade média foi, par excellence, a ferramenta fundamental para o uso da astronomia grega. necessários para determinar a posição dos planetas, do Sol e da Lua. Este livro permitiu usar de forma prática a teoria do Almagesto. O terceiro livro tem como título: Hipóteses Planetárias ${ }^{3}$ Neste livro Ptolomeu discute os princípios físicos de seu modelo geocêntrico e coloca de forma resumida o modelo matemático do Almagesto.

É interessante observar, do ponto de vista histórico, qual parte do Almagesto é uma contribuição original de Ptolomeu. No entanto, isto constitui uma questão complicada de resolver porque não se têm fontes históricas disponíveis, contemporâneas ao Almagesto, para fazer um trabalho de comparação. Uma referência próxima é a obra de Geminus chamada de Introduction Astronomiae, a qual deve ser do primeiro século [4], e se fundamenta na astronomia de Hiparco. Nesta obra Geminus não apresentar cálculos detalhados sendo uma obra mais qualitativa que quantitativa. Por outro lado, pesquisas recentes parecem coincidir que a principal contribuição original de Ptolomeu foi a introdução do ponto equante [6,7]. O ponto equante foi um ponto imaginário com respeito ao qual a rotação do planeta era constante. Isto permitiu seguir mantendo, de alguma forma, o conceito de rotação constante e acomodar movimentos irregulares vistos desde a Terra.

Posteriormente, o Almagesto encontrou uma grande recepção na cultura árabe, especialmente, na época do califa Al-ma'mun (786 - 833 d.C.) ${ }^{4}$ quem impulsionou à "Casa da sabedoria"em Bagdad. Al-ma'mun mandou construir um observatório para refazer as observações do Almagesto. Para isso, reuniu uma grande quantidade de astrônomos da época vindos de diferentes partes do mundo. Isto pode ser considerado como uma das primeiras iniciativas do estado para financiar um grande projeto de pesquisa científica. Assim, no 828 d.C os astronômos Yahya ibn abi Mansur e Sanad ibn Ali al-Alyahudi foram os encargados do projeto de Al-ma'mun [8]. A primeira versão do Almagesto em árabe também data desta época. Os resultados destes estudos foram compilados e publicados em um trabalho no 832 d.C. chamado de "Tábuas Verficadas" 9].

Por outro lado, as primeiras versões do Almagesto ao latim surgem no século XII, em Toledo, com o trabalho do tradutor Gerardo de Cremona (1114-1187). Ele foi um dos mais famosos tradutores da escola de tradutores de Toledo. Esta escola de tradutores no século XIII foi impulsionada pelo rei Alfonso X (1221-1284), o sabio. Nesta escola se traduziu ao castelhano muitas obras científicas do árabe e do grego. O fato de preferir o castelhano ao latim nas traduções foi fundamental para a formação da lingua castelhana. O rei Alfonso foi um grande mecenas da ciência e organizou um grande centro

\footnotetext{
${ }^{3}$ Igual ao Handy Tables é um livro posterior ao Almagesto, que trata a teoria geocêntrica de forma concisa. Na primeira tradução do grego para o latim, em 1620, o acadêmico John Bainbridgeno comentou o fato de Ptolomeu apresentar uma teoria de latitude diferente à teoria apresentada no Almagesto.

${ }^{4}$ Uma cópia do Almagesto foi uma das condições para fechar a paz com o Imperio Romano do Oriente.
} 
de estudos astronômicos em Toledo. Mandou refazer as conhecidas tábuas toledanas do século XI, as quais tinham sido organizadas pelo famoso astrônomo Arzaquel 5 Os encargados para organizar as novas tábuas foram os astronômos, de origem Judaica, Yehuda ben Moshe e Isaac ben Sid. Estas novas tábuas foram conhecidas como as Tábuas Afonsinas, as quais se converteram nas tábuas astronômicas padrão, usadas em diferentes partes da Europa. Por exemplo, Tycho Brahe ( 1546-1601) no século XVI ainda usava uma versão destas tábuas em seus estudos de astronomia.

Com estas traduções e tábuas astronômicas o modelo geocêntrico do Almagesto entra completamente ao ocidente. Comentadores e críticos do modelo de Ptolomeu surgem especialmente durante o século XIV. Em torno do ano de 1320 as Tábuas Afonsinas são estudadas em Paris e traduzidas do castelhano para o latim. É famosa a versão feita pelo famoso astrônomo, Johannes de Saxonia (1323-1355) (também chamado John of Saxony), quem adicionou instruções e comentários para seu uso. Este académico desenvolveu seu trabalho na Universidade de Paris, na primeira metade do século XIV. Esta versão das Tábuas Afonsinas em latim permitiu que elas se espalhassem na europa medieval. Adicionalmente, as tábuas foram refeitas para seu uso de acordo com o meriadiano do lugar.

Posteriormente, é importante destacar o trabalho de Georg von Peuerbach (1423-1461), que entre outras contribuições, escreveu um texto com o objetivo de ser usado no ensino universitário conhecido como Theoricae Novae Planetarum para apresentar de forma pedagógica o texto do Almagesto. Seu trabalho foi continuado por seu discípulo Johannes Müller von Königsberg (1436-1476) conhecido como Regiomontanus, que escreveu um livro chamado: Epítome do Almagesto, publicado no ano de 1496, que se transformou num livro texto muito popular para o ensino da astronomia da época. Copérnico (14731543) foi um dos leitores do Epítome do Almagesto e a influência sobre seu primeiro livro chamado Commentariolus, publicado aproximadamente no ano de 1515, é bastante notável. Para estudos recentes sobre o tema, ver referência $10^{6}$.

Por outro lado, a obra-mestra de Copérnico é o livro chamado De Revolutionibus Orbium Coelestium. Na primeira parte do livro, Copérnico apresenta uma crítica ao modelo geocêntrico e mostra seu interesse em voltar aos antigos moldes gregos estabelecidos por Platão, isto é, salvar os fenômenos 11. Para Copérnico o Almagesto não seguia estritamente as ideias de Platão, pois entre suas ferramentas estava o mencionado ponto equante. Copérnico procurou um método alternativo. Este método

\footnotetext{
${ }^{5}$ Nome latinizado do astrônomo Al-andalus, nascido em Toledo, de nome Abu Ixaque Ibraim al-Zarcali. Foi muito influente na sua época.

${ }^{6} \mathrm{Na}$ referência citada 10 o autor, Swerdlow, mostra que usando o livro Epítome do Almagesto é possível derivar uma teoria heliocêntrica da segunda desigualdade da anomalia dos planetas superiores e inferiores.
}

resultou em colocar a Terra em movimento. Assim, no De Revolutionibus, Copérnico tentou justificar o movimento da Terra e contestou os argumentos aristotélicos da época 7 .

Neste artigo, discutimos unicamente o movimento genérico dos planetas sem considerar o movimento do Sol e da Lua, e sem mostrar as diferenças detalhadas entre o movimento de planetas exteriores (Marte, Júpiter e Saturno) e interiores (Vênus e Mercúrio). Desta forma, na seção II apresentamos o modelo de Ptolomeu com suas principais características e as relações matemáticas usadas para determinar a longitude dos planetas. Também citamos, de forma breve, considerações sobre o cálculo das latitudes. Na seção III, discutimos a equivalência cinemática entre os modelos de Ptolomeu e de Copérnico. Adicionalmente, na seção IV, discutimos algumas características sobre a transição entre o modelo geocêntrico de Ptolomeu e o modelo heliocêntrico. Na seção V, apresentamos as conclusões.

\section{Longitude no modelo de Ptolomeu}

Para determinar a longitude de um planeta, Ptolomeu, considerou o movimento deste sobre um círculo denominado de epiciclo, cujo centro se movimentava sobre outro círculo maior chamado deferente. Ptolomeu considerou que o deferente é um círculo excêntrico e adicionou um novo ponto chamado de ponto equante com respeito ao qual a velocidade de rotação do planeta resultava constante. Como dito anteriormente, com o uso do equante, Ptolomeu, tentou manter tanto a ideia platônica de velocidade constante quanto construir um modelo que concorde com as observações astronômicas. Desde o ponto de vista moderno a introdução do equante pode ser pensado como um ajuste fino sobre a teoria. Porém, a ideia do equante resultou conflitiva ao longo dos séculos. Por exemplo, os árabes tentaram voltar ao modelo das esferas concêntricas de Eudoxo como alternativa ao uso do equante e, de forma similar, Copérnico, demonstra sua discordância com o uso do equante no inicio do De Revolutionibus [11.

As principais características geométricas do modelo de Ptolomeu são mostradas na Figura 1. O ponto $E$ representa o ponto equante. $O$ ponto $T$ representa a posição da Terra a qual está deslocada do centro do deferente, dado pelo ponto $D$, por uma distância $e$. O segmento $\overline{T A}$ define a linha apsidal, sendo que $A$ é denominado de apogeu, isto é, quando o centro do epiciclo $C$ passa por esse ponto o planeta está o mais afastado possível da Terra. $\mathrm{Na}$ antiguidade a linha $\overline{T A}$ era usada como referência. A linha que une o centro do epiciclo com a Terra é $\overline{T C}$. O ponto equante está afastado do centro do deferente na mesma distância que a Terra. Portanto, entre a Terra e o equante se define uma distância $2 e$, onde $e$ é a excentricidade. $\mathrm{O}$ ângulo $\lambda$ é denominado de longitude, o ângulo $\lambda_{m}$ é a longitude média, ambos ângulos são medidos com

${ }^{7} \mathrm{Na}$ época de Copérnico as leis do movimento eram entendidas do ponto de vista Aristotélico. 


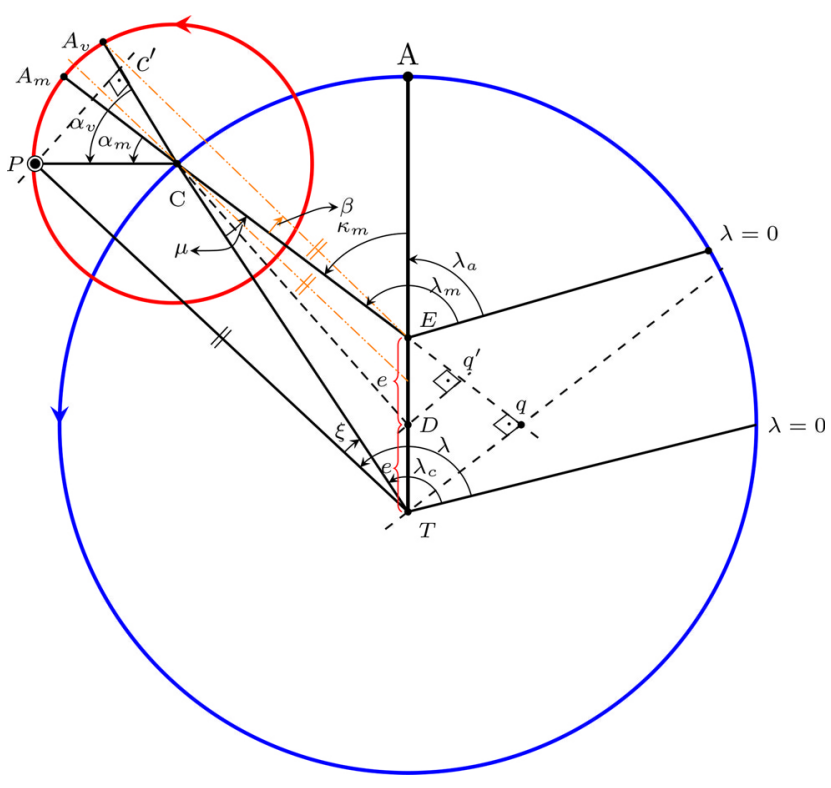

Figura 1: Principais elementos geométricos do modelo de Ptolomeu para determinar a Longitude. Para detalhes ver o texto.

respeito a uma linha arbitrária assinalada na figura como $\lambda=0$. Em geral, a posição desta linha dependerá do calendário usado como referência para fazer a contagem do tempo. Por exemplo, Ptolomeu utilizou o calendário da era de Nabonassar. ${ }^{8}$ Adicionalmente, sobre o epiciclo definimos dois ângulos que chamamos de $\alpha_{m}$, ângulo da anomalia média, definido pela linha de referência $A_{m}$ e o segmento $\overline{C P}$. O outro ângulo é o $\alpha_{v}$ que é o ângulo entre a linha de referência $A_{m}$ e o segmento $\overline{C P}$. O ponto $A_{v}$ é chamada de linha do apogeu verdadeiro, isto é, o apogeu com respeito à Terra.

Desde o ponto de vista geométrico, devido ao uso do ponto equante, é necessario alguns ângulos adicionais. Por exemplo, o ângulo entre os segmentos $\overline{E A}$ e $\overline{E C}$, $k_{m}$, é denominado ângulo do centro médio, o qual permite localizar o centro do epiciclo desde o equante, e resulta igual a diferença entre o ângulo $\lambda_{m}$ e o ângulo $\lambda_{a}$, chamado de longitude do apogeu. Também é necessario o ângulo $\mu$ formado pelos segmentos $\overline{E C}$ e $\overline{T C}$, denominado prostaphairesis da anomalia. Note-se que se as medidas são feitas diretamente da Terra, sem considerar o equante, não teriamos o ângulo $\mu$. Este ângulo depende fundamentalmente da medida do ângulo do centro $k_{m}$ e do valor da distância entre a Terra e o ponto equante, isto é, o segmento $\overline{T E}$. Adicionalmente, também dependerá do valor do raio do deferente $R$, pois quando maior seja o raio menor será o ângulo subtendido da relação $s=R \theta$, onde $s$ é o arco de circunferência. Também observando a figura podemos ver que, $\xi$, é o ângulo entre os segmentos $\overline{T P}$ e $\overline{T C}$, denominado de prostaphairesis da longitude.

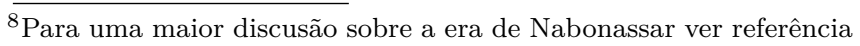
4
Estes são os principais ângulos usados no modelo de Ptolomed 9

Se observamos a Figura 1, podemos ver, por uma simples construção geométrica, que a longitude $\lambda$ pode ser determinada em geral da seguinte forma: coloquemos uma reta paralela ao segmento $\overline{T P}$ passando pelo ponto $C$ e outra paralela, também ao segmento $\overline{T P}$, passando pelo equante $E$. Com esta construção geométrica podemos usar el teorema de ângulos iguais entre retas paralelas para escrever,

$$
\lambda=\lambda_{m}-\beta,
$$

onde o ângulo $\beta$ é o ângulo entre a reta paralela que passa pelo equante e a reta $\overline{C E}$. Para determinar este ângulo podemos construir uma reta passando pelo centro do epiciclo, ponto $\mathrm{C}$, paralelo ao segmento $\overline{T P}$ (essa paralela em geral não coincide com o segmento $\overline{D C}$ ). Assim, observando esta contrução podemos ver que o ângulo entre a reta paralela que passa pelo ponto $C$ e o segmento $\overline{T C}$ é $\xi$, assim temos:

$$
\beta=\mu-\xi
$$

Portanto, podemos escrever em forma geral que a longitude do planeta pode ser escrito como:

$$
\lambda=\lambda_{m}-\mu+\xi
$$

Agora vamos a determinar o ângulo $\mu$ para o qual utilizamos a lei dos senos sobre o triângulo $\triangle C T E$ de forma que é possível escrever,

$$
\mu=\sin ^{-1}\left(\frac{2 e \sin \left(k_{m}\right)}{\overline{T C}}\right),
$$

Por outro lado, podemos facilmente determinar o ângulo $\xi$ usando o triângulo $\triangle P C T$ :

$$
\xi\left(\alpha_{v}, k_{m}\right)=\sin ^{-1}\left(\frac{r \sin \left(\alpha_{v}\right)}{\overline{T P})}\right),
$$

onde $r$ representa o raio do epiciclo e o ângulo $\alpha_{v}$, como mencionado é o ângulo entre a linha $\overline{C A_{v}}$ e a linha $\overline{C P}$. Por outro lado, para determinar a distância $\overline{T P}$ podemos fazer uso do triângulo retângulo $\triangle T P c^{\prime}$, assim temos,

$$
\overline{T P}=\sqrt{\left(\overline{T C}+r \cos \left(\alpha_{v}\right)\right)^{2}+\left(r \sin \left(\alpha_{v}\right)\right)^{2}} .
$$

Como podemos observar para poder definir adequadamente as expressões anteriores é necessario determinar a distância $\overline{T C}$, para isso, observando a Figura 1. podemos considerar o triângulo retângulo $\triangle T C q$ e escrever:

$$
\overline{T C}^{2}=\overline{T q}^{2}+\overline{q C}^{2} .
$$

Agora para determinar o lado $\overline{T q}$ podemos considerar a semelhança entre os triângulo $\Delta E D q^{\prime}$ e $\Delta E T q$. Desta

\footnotetext{
${ }^{9}$ Neste artigo usamos funções trigonométricas e equações algébricas. Ptolomeu não usou estas funções e equações no Almagesto.
} 
forma se, nesses triângulos, o ângulo $E$ é denotado como $\theta$ podemos escrever,

$$
\sin \theta=\frac{\overline{T q}}{2 e} \Longrightarrow \overline{T q}=2 e \sin \theta .
$$

Para o lado $\overline{q C}$ podemos observar na figura que

$$
\overline{q C}=\overline{C q^{\prime}}+\overline{q^{\prime} q} .
$$

De outro lado, se consideramos o triângulo $\Delta C D q^{\prime}$ e que $R=\overline{C D}$, então é possível fazermos a seguinte análise,

$$
\begin{aligned}
R^{2} & ={\overline{C q^{\prime}}}^{2}+{\overline{q^{\prime} D}}^{2} \quad \text { onde } \quad \overline{q^{\prime} D}=e \sin \theta, \\
\overline{C q^{\prime}} & =\left(R^{2}-e^{2} \sin ^{2} \theta\right)^{1 / 2} .
\end{aligned}
$$

Para completar a equação (7) resta o lado $\overline{q q^{\prime}}$ que por semelhança de triângulos podemos estabelecer que $\overline{q q^{\prime}}=\overline{E q^{\prime}}=e \cos \theta$, assim temos que, substituindo todos estes resultados na equação (7) obtemos,

$$
\overline{T C}^{2}=4 e^{2} \sin ^{2} \theta+\left\{\left(R^{2}-e^{2} \sin ^{2} \theta\right)^{1 / 2}+e \cos \theta\right\}^{2} .
$$

No entanto, o ângulo $\theta$ da Figura 1 é oposto e igual ao ângulo $k_{m}$, e portanto, temos que: $k_{m}=\theta$. Assim, podemos observar que o valor do lado $\overline{T C}$ depende explicitamente do ângulo $k_{m}$ como,

$$
T C\left(k_{m}\right)=\sqrt{\left[\sqrt{R^{2}-\left(e \sin \left(k_{m}\right)\right)^{2}}+e \cos \left(k_{m}\right)\right]^{2}+\left(2 e \sin \left(k_{m}\right)\right)^{2}} .
$$

Agora considerando a localização do planeta dentro do epiciclo mediante os dois ângulos $\alpha_{v}$ e $\alpha_{m}$, é fácil ver que o ângulo da anomalia verdadeira $\alpha_{v}$ é igual a soma do ângulo da anomalia média mais o ângulo $\mu$ :

$$
\alpha_{v}=\alpha_{m}+\mu\left(k_{m}\right) .
$$

Portanto, podemos usar todos estes resultados para determinar a longitude de um planeta pela equação (3), escrevendo explicitamente a dependência angular, isto é,

$$
\lambda=\lambda_{m}-\mu\left(k_{m}\right)+\xi\left(\alpha_{v}, k_{m}\right) .
$$

Assim, podemos usar este resultado para determinar a longitude de um planeta. No modelo de Ptolomeu o período de rotação do centro do epiciclo, em torno do centro do deferente, corresponde com o período de revolução do planeta. Adicionalmente, o período de revolução do epiciclo deve coincidir com o tempo entre duas retrogradações. Sem entrar em detalhes de como Ptolomeu fazia medidas do tempo, isto é, como contava os anos vamos a assumir alguns valores para as constantes $R, r$, e podemos variar os ângulos $k_{m}$ e $\lambda_{m}$ para determinar a longitude do planeta. Por exemplo, vamos a assumir alguns valores usados no Almagesto: para o planeta Saturno: $R=60$, $r=6.5$. Adicionalmente, como anteriormente dito, consideramos: $\mu=\lambda_{m}-\lambda_{a}$, onde $\lambda_{a}$ é a longitude do apogeu.
Assim, podemos construir o gráfico de $\mu$ com respeito a longitude média. Isto é mostrado na Figura 2, onde também fazemos os gráficos de $\xi$ como função do ângulo $\alpha_{m}$ e da longitude $\lambda$ também como função de $\alpha_{m}$ para o qual fixamos o valor de $\lambda_{m}$.

\section{Latitude no modelo de Ptolomeu}

Para determinar a latitude dos planetas Ptolomeu não desenvolveu um único método, ele reformulou seu esquema de cálculo da latitude em três oportunidades: no Almagesto, nas Tábuas Práticas e nas Hipóteses Planetárias.
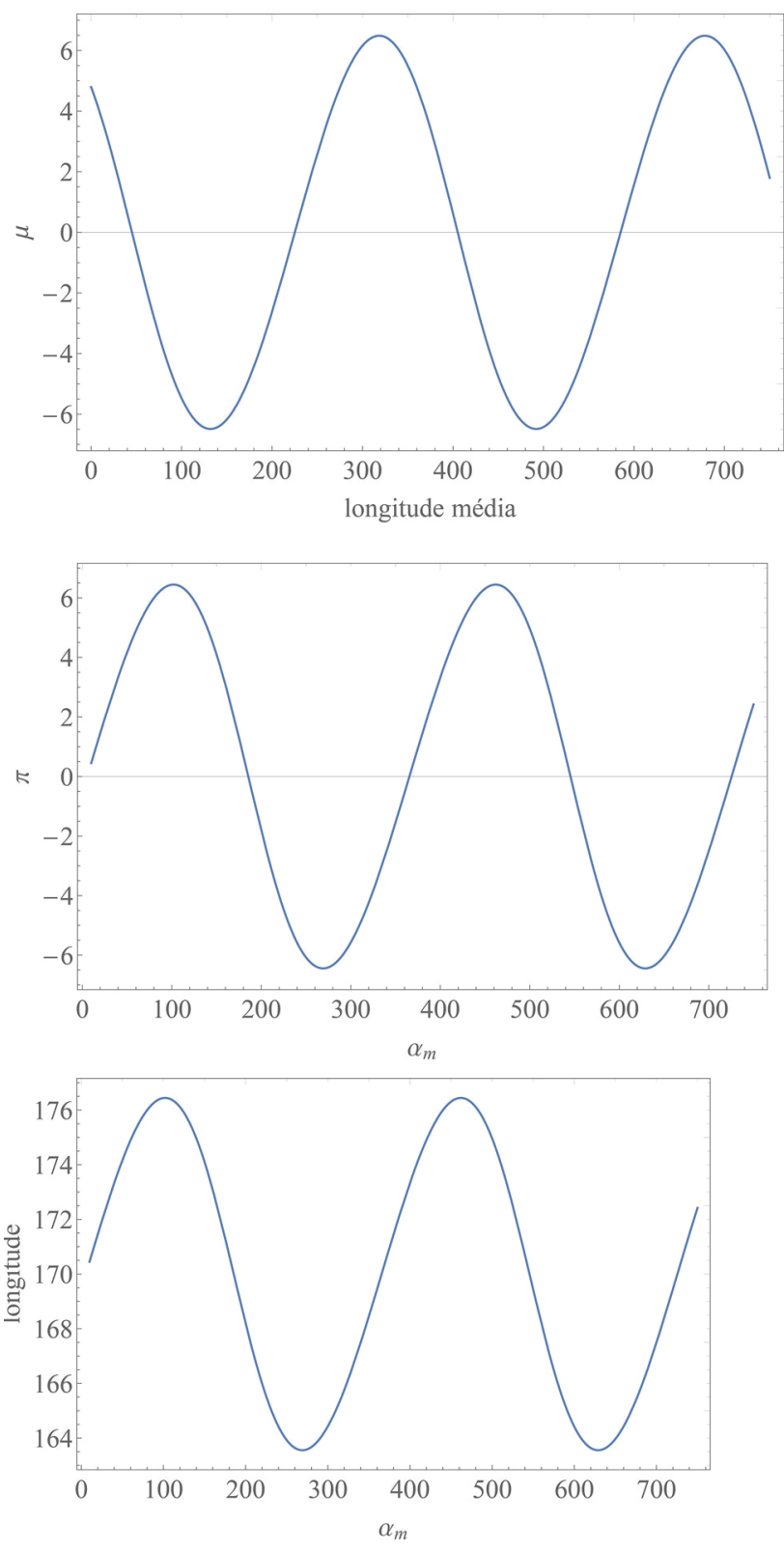

Figura 2: Na primeira figura mostramos a variação do ângulo $\mu$ como função da longitude média $\lambda_{m}$ para o planeta Saturno. Assumimos como longitude do apogeo o valor de $225.2^{\circ}$. Para fazer o gráfico de $\xi$ e $\lambda$ assumimos como valor fixo de longitude média $\lambda_{m}=165^{\circ}$ e variamos o ângulo $\alpha_{m}$. 
Neste trabalho apresentamos o método do Almagesto, o qual constitui o método padrão, sendo as outras modificações desta. O método das latitudes de Ptolomeu é basicamente empírico, sem chegar a construir um método geométrico consistente como no caso do cálculo da longitude. Em todos os casos, a ideia geral inclui uma inclinação do deferente em relação ao plano da eclíptica, e uma inclinação do plano do epiciclo em relação ao deferente, de forma que os planos do epiciclo e da eclíptica sejam paralelos.

Para os três planetas superiores foi assumido uma inclinação do deferente com respeito à eclíptica de $1^{\circ}$ para Marte, de $1^{\circ} 30^{\prime}$ para Júpiter e de $2^{\circ} 30^{\prime}$ para Saturno. Para Marte a linha de apsides do deferente foi colocada perpendicular à linha de interseção entre os planos do deferente e a eclíptica. A linha das apsides coincide com a linha norte-sul. Para Júpiter esta linha norte-sul faz $20^{\circ}$ ao oeste e para Saturno $50^{\circ}$ também com respeito ao oeste 12 .

De outro lado, para os planetas interiores, Vênus e Mercúrio, o ângulo entre os planos do deferente e da eclíptica, deveria ser oscilante, entre os límites de 10' para Vênus e de 45' para Mercúrio [12].

O cálculo da latitude permaneceu como um dos principais problemas a ser resolvidos dentro do modelo. Nesta questão o modelo de Copérnico era técnicamente superior, entre outras coisas, por assumir a variação do ângulo em relação ao Sol e não à Terra. No entanto, a questão de determinar a latitude só vai ficar relativamente consistente na época de Kepler [13].

\section{Equivalência Cinemática dos Modelos de Ptolomeu e Copérnico}

Nesta seção vamos a mostrar a equivalência geométrica entre os modelos de Copérnico e Ptolomeu. O modelo de Copérnico determina o movimento dos planetas com respeito ao Sol médio, ou seja, em relação ao centro da órbita da Terra, posição que não coincide com a posição do Sol. Adicionalmente, Copérnico introduz um pequeno epiciclo, chamado de epicicleto, o qual gira em sentido contrário ao epiciclo de Ptolomeu.

Para mostrar a equivalência entre os modelos vamos a usar a contrução geométrica mostrada na Figura 3, na qual o Sol médio está representado pela letra $\bar{S}$ do círculo tracejado com centro em 0 . Na mesma figura um planeta está representado pela letra $P$ e se movimenta em um pequeno epicicleto de centro $C$. Para mostrar a equivalência resulta útil usar o círculo sólido azul. Vamos agora a construir um paralelograma de lados paralelos $\overline{O^{\prime} O}$ e $\overline{C^{\prime} C}$. De estas considerações podemos observar, da Figura 3, que o círculo com centro em $C^{\prime \prime}$ pode ser considerado como um epiciclo. Então, para obtermos o modelo de Ptolomeu, desde o modelo tracejado de Copérnico, é necessario encontrar um círculo deferente para o epiciclo com centro em $C^{\prime \prime}$. Este círculo deferente é o círculo sólido amarelo.

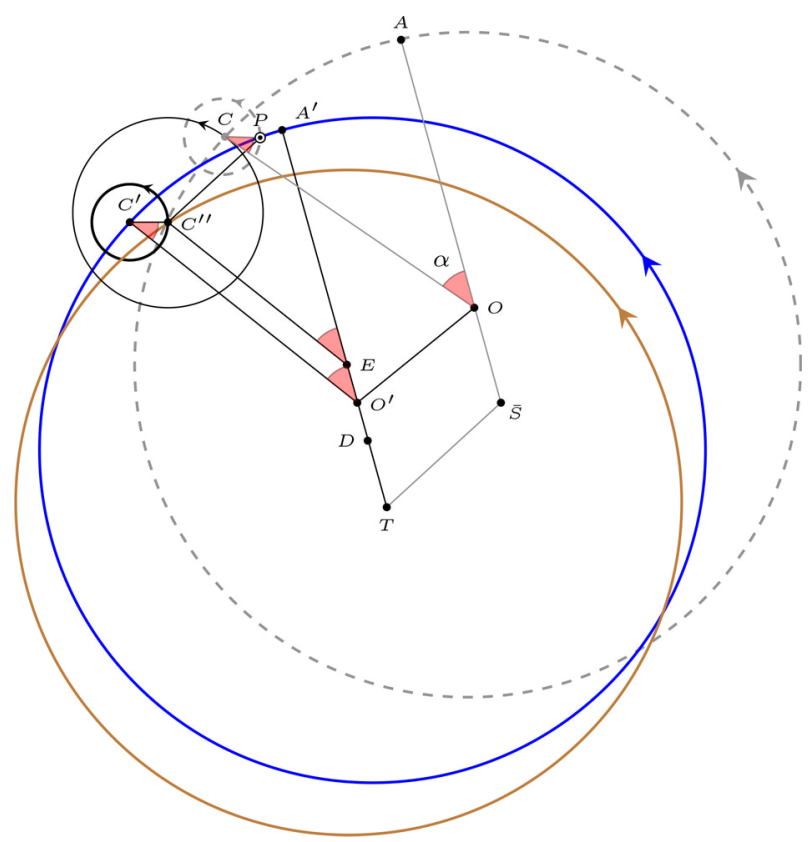

Figura 3: Apresentamos a construção geométrica usada para mostrar a equivalência entre o modelo de Ptolomeu e o modelo de Copérnico.

Para explicitamente mostrar a equivalência entre os modelos de Copérnico e Ptolomeu, podemos determinar a posição do planeta, com respeito à Terra por duas trajetórias diferentes: a primeira seguindo o modelo de Copérnico (isto é seguindo a trajetoria no circulo tracejado) e a segunda seguindo o modelo de Ptolomeu (isto é seguindo a trajetória da linha sólida). Para um maior detalhe mostramos estas duas trajetórias na Figura 4 Então, para ambos os casos, usando estas figuras podemos determinar as coordenadas do planeta em suas componentes cartesianas. Para fazer isto estabelecemos que o eixo $Y$ é definido pela linha das apsides, $A$ ou $A^{\prime}$, assim temos:

$$
\begin{aligned}
X_{\text {cop }} & =\overline{T \bar{S}} \cos (\theta)-\overline{O C} \cos (\alpha) \\
& +\overline{C P} \cos (\phi), \\
Y_{\text {cop }} & =\overline{T \bar{S}} \sin (\theta)+\overline{\bar{S} O}+\overline{O C} \sin (\alpha) \\
& +\overline{C P} \sin (\phi),
\end{aligned}
$$

onde de acordo com a Figura 4 o ângulo $\theta$ é o ângulo no vértice $\bar{S}$, o ângulo $\alpha$ corresponde ao ângulo no vértice $O$ e o ângulo $\phi$ ao ângulo no vértice $C$. Para o modelo de Ptolomeu obtemos as coordenadas,

$$
\begin{aligned}
X_{\text {pto }} & =-\overline{O^{\prime} C^{\prime}} \cos \left(\alpha^{\prime}\right)+\overline{C^{\prime} C^{\prime \prime}} \cos \left(\phi^{\prime}\right) \\
& +\overline{C^{\prime \prime} P} \cos \left(\theta^{\prime}\right), \\
Y_{\text {pto }} & =\overline{T O^{\prime}}+\overline{O^{\prime} C^{\prime}} \sin \left(\alpha^{\prime}\right)+\overline{C^{\prime} C^{\prime \prime}} \sin \left(\phi^{\prime}\right) \\
& +\overline{C^{\prime \prime} P} \sin \left(\theta^{\prime}\right) .
\end{aligned}
$$

onde, novamente pela Figura 4, o ângulo $\theta^{\prime}$ é o ângulo no vértice $C^{\prime \prime}$, o ângulo $\alpha^{\prime}$ corresponde ao ângulo no 

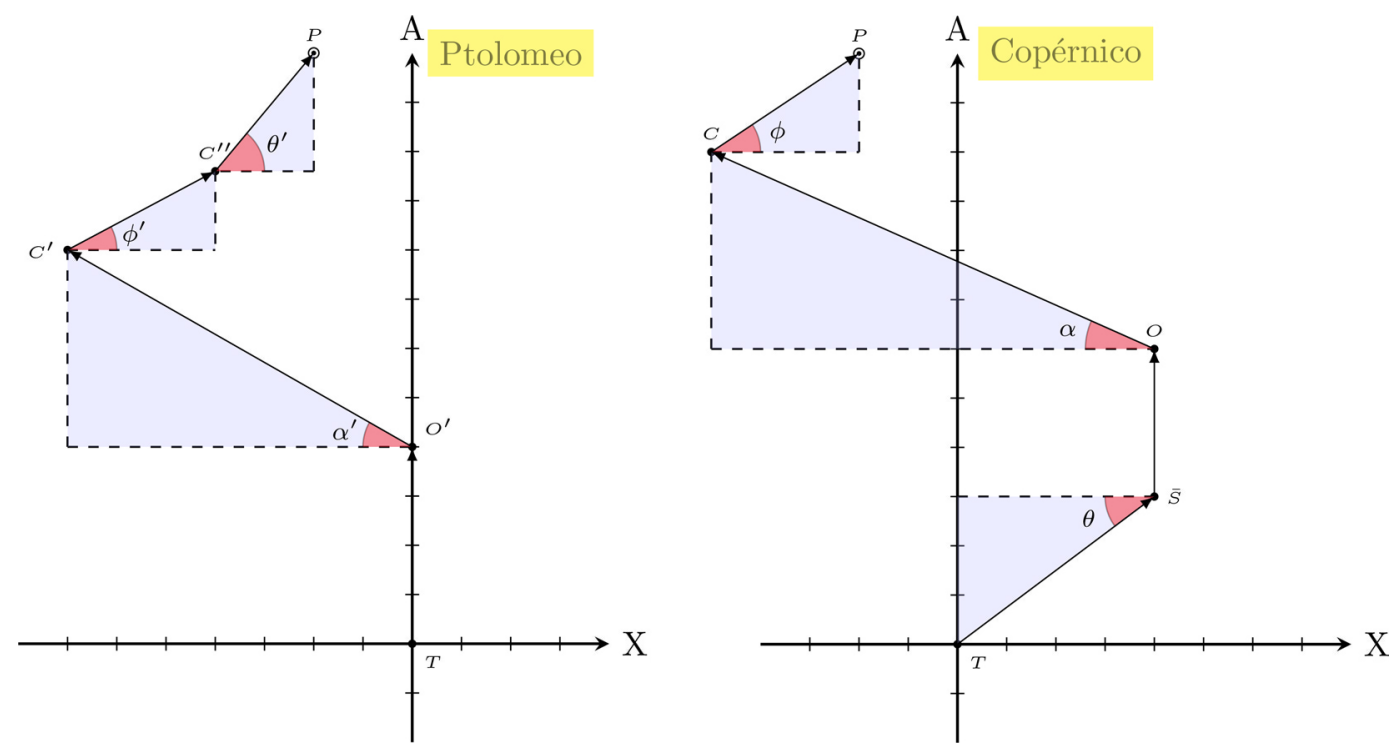

Figura 4: Na figura da esquerda mostramos a trajetória seguida desde a Terra até o planeta de acordo com Ptolomeu. Na figura da direita mostramos a trajetória para o modelo de Copérnico. O eixo $Y$ é paralelo aos eixos $A^{\prime}$ e $A$.

vértice $O^{\prime}$ e o ângulo $\phi^{\prime}$ ao ângulo no vértice $C^{\prime}$. Agora pela construção geométrica do paralelograma da Figura 3 temos que os segmentos $\overline{C^{\prime} O^{\prime}}$ e $\overline{C O}$ são parelelos e os eixos $A^{\prime}$ e $A$ também, assim, concluimos que $\alpha^{\prime}=\alpha$. Ademais, como os segmentos $\overline{T \bar{S}}$ e $\overline{C^{\prime \prime} P}$ são paralelos (a figura neste caso não está a escala), também podemos inferir que $\theta^{\prime}=\theta$. Adicionalmente, temos que os segmentos $\overline{C P}$ e $\overline{C^{\prime} C^{\prime \prime}}$ são iguais e paralelos (neste caso a figura também não está a escala), portanto, obtemos $\phi^{\prime}=\phi$. Em forma resumida temos por construção:

$$
\begin{aligned}
\overline{T O^{\prime}} & =\overline{\bar{S} O}, \\
\overline{O^{\prime} C^{\prime}} & =\overline{O C}, \\
\overline{C^{\prime} C^{\prime \prime}} & =\overline{C P}, \\
\overline{C^{\prime \prime} P} & =\overline{T \bar{S}} .
\end{aligned}
$$

Substituindo estas igualdades nas coordenadas cartesianas de Ptolomeu e Copérnico e com as condições mencionadas anteriormente podemos concluir que:

$$
\begin{gathered}
X_{\text {cop }}=X_{p t o} \\
Y_{\text {cop }}=Y_{p t o} .
\end{gathered}
$$

Para completar nossa equivalência vamos a mostrar quais devem ser as características do deferente do modelo de Ptolomeu. Como se pode observar é fudamental manter a estrutura do paralelograma, esta é a condição para considerar a equivalência. Podemos ver da Figura 3 que o ângulo $\theta$ pode ser representado como: $\delta=\omega t$, onde $\omega$ é a velocidade ângular constante. Se mantemos a estrutura em paralelograma, então o ângulo no ponto $E$ será igual ao ângulo $\delta$ da figura. Portanto, o movimento do centro do epiciclo, ponto $C^{\prime \prime}$, é visto desde o ponto $E$ como girando com velocidade constante e este ponto pode ser considerado como o ponto equante no modelo de Ptolomeu. De outro lado, consideramos a posição inicial do movimento. Esta posição inicial é quando o planeta, isto é, o ponto $C^{\prime \prime}$, está sobre a linha apsidal, como mostrado na Figura 5, onde colocamos o centro do epiciclo $C^{\prime \prime}$ sobre a linha apsidal $\overline{T A^{\prime}}$. Vamos impor que o raio do deferente do modelo de Ptolomeu e do modelo de Copérnico sejam iguais a $R$. Assim, considerando o centro do círculo no ponto $D$, de acordo com a Figura 5, podemos escrever este raio de Ptolomeu como:

$$
R=\overline{D O^{\prime}}+\overline{O^{\prime} E}+\overline{E C^{\prime \prime}} .
$$

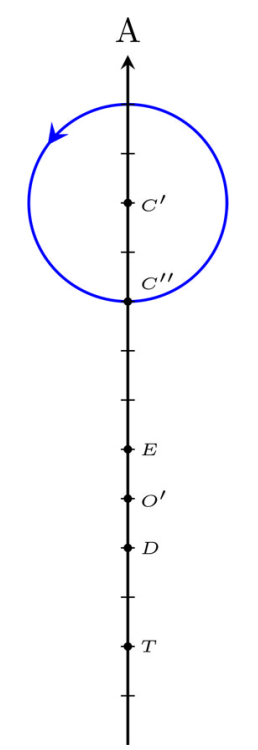

Figura 5: Mostramos a posição inicial sobre a linha apsidal, $A$, para construir a equivalência entre os modelos de Ptolomeu e Copérnico. 
Também, se consideramos o centro $O^{\prime}$, então no caso de Copérnico podemos escrever:

$$
R=\overline{O^{\prime} E}+\overline{E C^{\prime \prime}}+\overline{C^{\prime \prime} C^{\prime}} .
$$

Tanto a equação de $R$ para o caso de Ptolomeu, como para o caso de Copérnico, são considerados como iguais, porque constituiu a condição para manter a contrução em forma de paralelograma, e portanto, para manter a equivalência entre os modelos. Se comparamos as equações para $R$, podemos concluir que, $\overline{C^{\prime \prime} C^{\prime}}=\overline{D O^{\prime}}$. Adicionalmente, observando a Figura 3, podemos ver que as linhas $\overline{C^{\prime \prime} E}$ e $\overline{C^{\prime} O^{\prime}}$ são paralelas, e portanto, os segmentos de reta $\overline{C^{\prime \prime} C^{\prime}}$ e $\overline{O^{\prime} E}$ são iguais. Assim, de argumentos de geometria elemental, temos que: $\overline{O^{\prime} E}=\overline{D O^{\prime}}$. Por outro lado, lembrando que o ponto $D$ é o ponto central do segmento $\overline{T E}$, podemos escrever a seguinte igualdade:

$$
R=\frac{1}{2} \overline{T E}-\overline{D O^{\prime}}-\overline{C^{\prime \prime} C^{\prime}}+R,
$$

pois, acabamos de mostramos que $\overline{C^{\prime \prime} C^{\prime}}=\overline{O^{\prime} E}$. Destes resultados e novamente da Figura 3, podemos escrever que,

$$
\overline{C P}=\overline{D O^{\prime}}=\overline{C^{\prime \prime} C^{\prime}},
$$

assim, podemos reescrever em função de $\overline{C P}$,

$$
R=\frac{1}{2} \overline{T E}-2 \overline{C P}+R .
$$

Colocando em evidência $\overline{T E}$ da equação anterior, obtemos,

$$
\overline{T E}=4 \overline{C P},
$$

considerando a condição para mater o paralelogramo $T O^{\prime} O \bar{S}$ podemos escrever,

$$
\overline{T E}=\overline{T O^{\prime}}+\overline{O^{\prime} E}=\overline{\bar{S} O}+\overline{C P} .
$$

Portanto, se substituímos a equação de $\overline{T E}$ podemos determinar o resultado:

$$
\overline{\bar{S} O}=3 \overline{C P},
$$

assim, desta forma mostramos que o modelo de Copérnico redistribui a excêntricidade de Ptolomeu, determinando para o raio do epicicleto, $\overline{C P}$, o valor de $\frac{\overline{\bar{S} O}}{3}$. Mas desde o ponto de vista cinemático o resultado de Ptolomeu em longitude é equivalente ao modelo de Copérnico. Porém, desde o ponto de vista da construção de uma teoria o modelo de Copérnico resulta mas interessante. Pois no modelo de Ptolomeu é preciso assumir que em todo o movimento planetário deve se considerar que os segmentos de reta, $\overline{T \bar{S}}=\overline{C^{\prime \prime} P}$, para ajustar o movimento do planeta ao movimento do Sol. No modelo de Copérnico isso não é necessário, pois é substituido pelo movimento da Terra em torno do Sol. Também desta forma Copérnico contornou tecnicamente o uso do equante, mas sem deixar de usar o conceito de excentricidade, que era fundamental, para fazer os cálculos necessários na determinação da posição do planeta.

\section{Precisão e epiciclos sobre epiciclos}

É comum assumir que a transição entre o modelo de Ptolomeu e o modelo de Copérnico ocorreu devido a um estado de crise do modelo de Ptolomeu. Nesta linha de interpretação é importante resaltar o influente trabalho de Thomas Khun em seu livro "A Estrutura das Revoluções Científicas",

Se a consciência da anomalia desempenha um papel na emergência de novos tipos de fenômenos, ninguém deveria surpreender-se com o fato de uma consciência semelhante, embora mais profunda, seja um pré-requisito para todas as mudanças de teoria aceitáveis. Penso que a esse respeito a evidência histórica é totalmente inequívoca. A astronomia ptolemaica estava numa situação escandalosa, antes dos trabalhos de Copérnico 14.

No entanto, parece interessante verificar se há, de fato, evidências históricas sobre a crise. Neste sentido, as pesquisas de Owen Gingerich são muito esclarecedoras [15. Por exemplo, para o planeta Marte ele verifica que os erros na determinação da longitude usando a teoria de Ptolomeu de acordo com as efemérides de Regiomontanus e Stoeffler, se aproximam a uns $5^{\prime}$. Por outro lado, Kepler usando os parâmetros de Copérnico, no prefácio de suas tábuas Rudolphinas, menciona que os erros para Marte ficavam entorno de 5' 7]. Outra evidência histórica são as anotações de Tycho, quem usou tanto as tábuas Afonsinas, assim como, as tábuas Prutênicas feitas usando o modelo de Copérnico. Para ambas tábuas Tycho comenta que os erros são similares.

Assim, só observando os erros na posição dos planetas se torna difícil escolher um dado modelo. A teoria de Ptolomeu durante o século XVI parece ser bastante consistente e mesmo escolásticos como Oresme ou Buridano, que usaram argumentos lógicos para discutir o movimento da Terra e as esferas celestes, concluíram que o movimento da Terra não era verdadeiro, e portanto, não houve uma rejeição direta ao modelo geocêntrico de Ptolomeu. O que se observou foi um crescente número de astrônomos construindo tábuas e escrevendo textos explicativos e críticos ao Almagesto. Mas isso era feito, pelo menos, desde o século IX com os árabes.

No De Revolutionibus, Copérnico tenta salvar os fenômenos, com a intenção estética de voltar aos ideais gregos. Por exemplo, no primeiro livro do De Revolutionibus, como anteriormente mencionado, Copérnico discute e defende a ideia da trajetória circular e do movimento constante como premisas fundamentais para descrever os movimentos dos planetas e dos corpos celestes. Copérnico então argumenta contra as ideias aristotélicas sobre a Terra. Ele enfatiza a questão do movimento natural. Em seu texto, de uma forma rudimentar, ele se aproxima do princípio do movimento relativo. O argumento dos aristotélicos consistia basicamente em mencionar que se um objeto fosse lançado da superfície da Terra, o objeto 
não chegaria ao lugar esperado, pois, durante o tempo no qual o objeto estivesse se deslocando, a Terra também o faria, e portanto, não poderíamos observar a queda da pedra conforme a conhecemos. Assim, o que se pode inferir, sem dúvida, é uma questão de estética e volta aos antigos moldes gregos por parte de Copérnico.

Porém, por que o trabalho de Copérnico recebeu bastante atenção por parte dos astrônomos da época? Entre outras coisas, podemos dizer que o trabalho de Copérnico é extremamente minucioso e técnico, por isso, seus escritos se tornaram uma fonte paralela ao Almagesto de Ptolomeo. O De Revolutionibus é um livro onde Copérnico detalha e ensina ao leitor como fazer os cálculos para determinar a posição dos planetas, da Lua e do Sol, com um método alternativo ao método matemático desenvolvido por Ptolomeu no Almagesto. Copérnico coloca a Terra em movimento e Ptolomeu considera seus cálculos respeito à Terra fixa. Na história da ciência desde a publicação do Almagesto os astrônomos não dispunham de um livro que ensinasse um método matemático alternativo ao Almagesto. Copérnico escreveu um livro que incluia uma análises matemática de praticamente todos os fenômenos celestes estudados, também matematicamente, no Almagesto. Por isso, não é de surpreender que pouco depois da publicação do De Revolutionibus, o astrônomo, Erasmus Reinhold publicou as primeiras efemérides usando a teoria de Copérnico conhecidas como Tábuas Prutênicas.

Por outro lado, outra ideia bastante mencionada na literatura, é o fato de pensar que o sistema de Ptolomeu evoluiu para um sistema de epiciclos sobre epiciclos, resultando em um modelo inviável do ponto de vista matemático. Por exemplo, imaginemos que para um dado planeta seja necessário cinco epiciclos, um dentro do outro, isto constituíria, sem o uso fluente da geometria analítica, uma estrutura geométrica complexa e seria difícil obter predições específicas. No entanto, do ponto de vista histórico não há evidência a favor do uso de epiciclos sobre epiciclos, ao contrário, as pequisas feitas por Gingerich, recalculando as Tábuas Afonsinas, indicam que as tábuas foram construídas usando o modelo de Ptolomeu do Almagesto com um epiciclo e um deferente [16].

Atualmente considerar epiciclos sobre epiciclos pode ser modelado com certa facilidade. Por ejemplo, se consideramos um epiciclo e um deferente, e colocando o sistema de referência no centro do deferente, podemos escrever em forma paramétrica o raio vetor da posição de um ponto sobre o epiciclo da forma:

$$
r(t)=x(t) \hat{i}+y(t) \hat{j}
$$

sendo que cada coordenada pode ser escrita como:

$$
\begin{aligned}
& x(t)=r_{1} \cos \left(\omega_{1} t\right)+r_{2} \cos \left(\omega_{2} t\right), \\
& y(t)=r_{1} \sin \left(\omega_{1} t\right)+r_{2} \sin \left(\omega_{2} t\right),
\end{aligned}
$$

onde $r_{1}$ é o raio do deferente e $r_{2}$ é o raio do epiciclo, $\omega_{1}$ e $\omega_{2}$ representam as velocidade de rotação do deferente e do epiciclo respectivamente. Se, por ejemplo, temos um deferente e dois epiciclos podemos escrever:

$$
\begin{aligned}
& x(t)=r_{1} \cos \left(\omega_{1} t\right)+r_{2} \cos \left(\omega_{2} t\right)+r_{3} \cos \left(\omega_{3} t\right), \\
& y(t)=r_{1} \sin \left(\omega_{1} t\right)+r_{2} \sin \left(\omega_{2} t\right)+r_{3} \sin \left(\omega_{3} t\right),
\end{aligned}
$$

onde $r_{3}$ e $\omega_{3}$ é o raio e a velocidade de rotação do segundo epiciclo. Na Figura 6 mostramos os gráficos das equações anteriores com um e dois epiciclos para diferentes combinações dos valores assumidos dos raios e velocidades angulares. Em particular é notável observar que um sistema de dois epiciclos, figura da direita, pode produzir trajetórias intrincadas. A matemática disponível do século XV teria muitas dificuldades em estudar e predizer posições de planetas usando sistemas de epiciclos sobre epiciclos.

Uma generalização direta para um número $n$ qualquer de epiciclos sobre epiciclos pode ser facilmente construída 17:

$$
r(t)=\sum_{i}^{n}\left[a_{i} \cos \left(\omega_{i} t\right) \hat{i}+a_{i} \sin \left(\omega_{i} t\right) \hat{j}\right] .
$$

Este resultado, em geral, pode ser considerado como um caso particular da definição da série de Fourier trigonométrica a qual pode ser expressada como:

$$
r(t)=\frac{a_{0}}{2}+\sum_{i=1}^{\infty}\left[a_{i} \cos \left(\frac{n \pi t}{L}\right)+b_{i} \sin \left(\frac{n \pi t}{L}\right)\right],
$$

onde $r(t)$ é uma função periódica de período $2 L$ ou seja $r(t+2 L)=r(t)$ e para associar-se à definição de velocidade angular podemos considerar que $\omega_{n}=\frac{2 n \pi}{L}$. Esta série trigonométrica pode ser facilmente generalizada para ser escrita usando funções complexas [18,

$$
r(t)=\sum_{-\infty}^{\infty} c_{n} e^{i \omega_{n} t}
$$

onde os coeficientes $c_{n}$ podem ser determinados pela expressão:

$$
c_{n}=\frac{1}{2 L} \int_{-L}^{L} r(t) e^{-i \omega_{n} t} d t .
$$

Con esta definição de serie de Fourier podemos reproduzir o desenho de quase qualquer figura ${ }^{10}$. Para fazer isto é possível proceder em sentido contrário: inicialmente determinamos as coordenadas ou pontos do contorno da figura que queremos desenhar. Depois, usamos estes pontos para encontrar os raios dos círculos $\left(a_{i}, b_{i}\right)$ e as velocidades de rotação, $\omega_{i}$. Portanto, com estes dados podemos construir os epiciclos.

\footnotetext{
${ }^{10}$ Para maiores detalhes matemáticos, assim como, um programa para implementar o uso das séries de Fourier para desenhar ver: https://youtu.be/MY4luNgGfms
} 

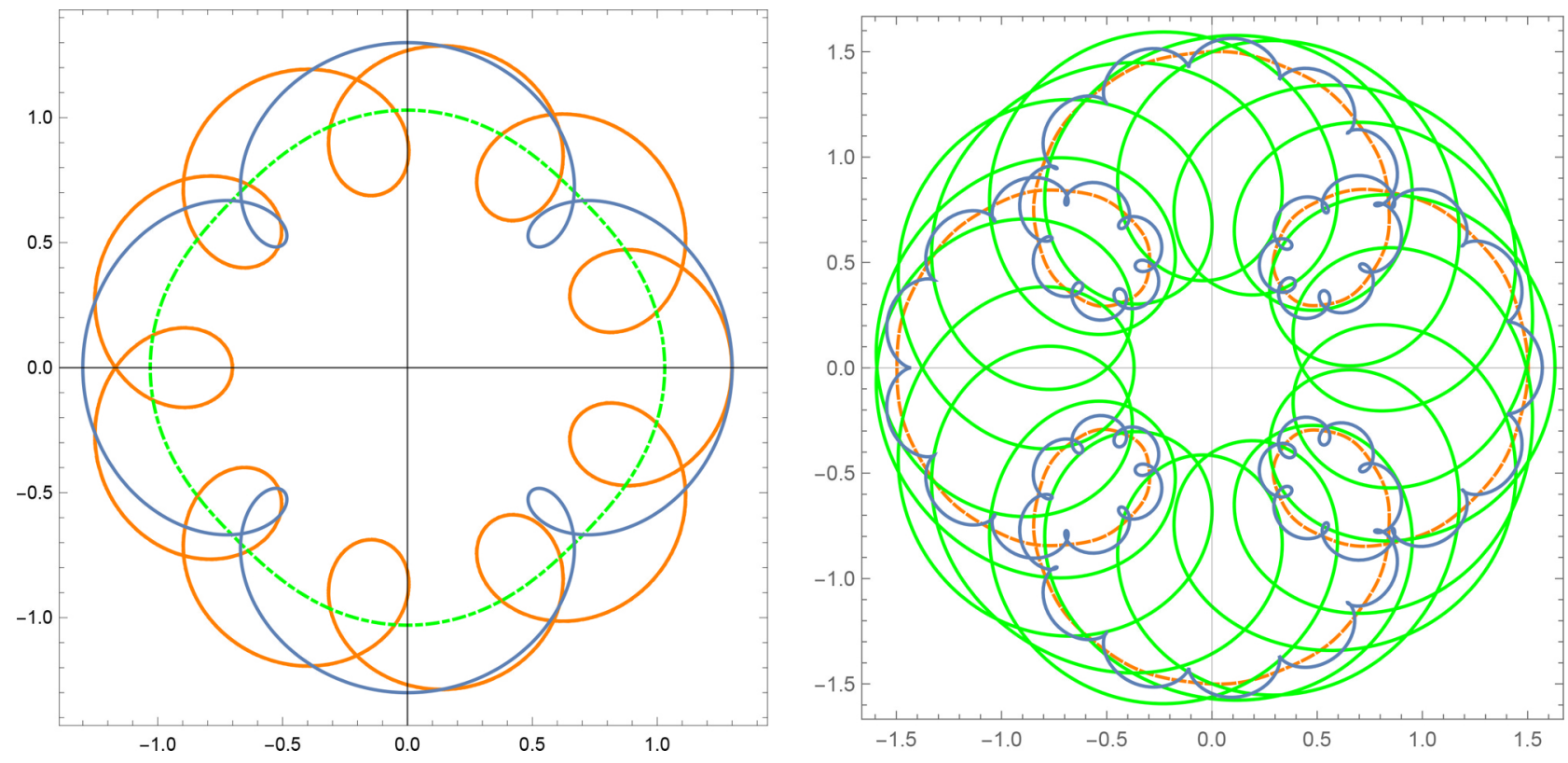

Figura 6: Na figura da esquerda mostramos o gráfico da trajetória produzido por um deferente e um epiciclo, onde a razão entre o raio do deferente e o epiciclo é $\frac{r_{e}}{r_{d}}=0.03$ (verde), $\frac{r_{e}}{r_{d}}=0.3$ (laranja e azul). A razão das velocidades $\frac{\omega_{d}}{\omega_{e}}=0.2$ (verde e azul), $\frac{\omega_{d}}{\omega_{e}}=0.1$ (laranja). No caso da figura da direita mostramos a trajetória produzida por um deferente e dois epiciclos. Os valores usados para construir os gráficos são: $r_{d}=1, r_{e 1}=0.5, r_{e 2}=0.003, \omega_{d}=2, \omega_{e 1}=10$ e $\omega_{e 2}=100$ (laranja); $r_{d}=1, r_{e 1}=0.5$, $r_{e 2}=0.07, \omega_{d}=2, \omega_{e 1}=10$ e $\omega_{e 2}=100$ (azul) e $r_{d}=1, r_{e 1}=0.6, r_{e 2}=0.03, \omega_{d}=3, \omega_{e 1}=20$ e $\omega_{e 2}=50$ (verde). Em todos os casos usamos o raio do deferente como unitário.

\section{Conclusões}

No presente artigo discutimos com uma linguagem matemática moderna as principais características do modelo de Ptolomeu. Especificamente mostramos como determinar a longitude de um planeta. Como nosso objetivo não é estudar o movimento de cada objeto celeste (dos cinco planetas, do Sol, da Lua e das estrelas fixas), consideramos um planeta exterior para apresentar os resultados. Esta escolha é conveniente porque praticamente todos os conceitos envolvidos na teoria de Ptolomeu; epiciclos, círculos excêntricos e equante são abordados para um planeta exterior (Marte, Jupiter e Saturno), mas com uma modelagem geométrica mais simples em comparação com um planeta interior. Porém, a metodologia é análoga. É interessante mencionar que por razões pedagógicas apresentamos todos os detalhes dos cálculos geométricos envolvidos.

Por outro lado, para o movimento em latitude, Ptolomeu assumiu valores empíricos e não desenvolveu uma teoria geométrica para derivar o valor da latitude como no caso da longitude. Ainda Ptolomeu apresenteou três versões diferentes de como determinar a latitude: no Almagesto, nas Tábuas Práticas e nas Hipóteses Planetárias. Estes resultados são comentados no texto.

Discutimos as condições necessárias para obtermos a equivalência geométrica entre os modelos de Ptolomeu e de Copérnico. Mostramos explicitamente esta equivalência desde o ponto de vista geométrico. Portanto, é esperado que ambos modelos tenham predições de longitude próximas. Isto pode ser verificado nos dados históricos, por exemplo, revisar referência [15]. Assim, tanto as efemérides pré-copérnico como as pós-copérnico apresentam erros aproximadamente iguais. Esta situação é diferente, por exemplo, com as Tábuas Rudolfinas de Kepler 15], onde uma nova ferramenta matemática é usada: a teoria da elipse, em geral, a teoria das cônicas ${ }^{11}$

Consideramos duas questões principais sobre a transição entre o modelo de Ptolomeu e o modelo de Copérnico: a primeira se refere à precisão na determinação das posições dos objetos celestes por cada uma das teorias. A segunda se refere ao suposto uso excessivo de epiciclos no modelo de Ptolomeu. Em cada caso mostramos argumentos relativamente simples do ponto de vista histórico, o que nos permite ter uma visão completa destas questões, muitas vezes, como mencionado no texto, erroneamente compreendidas.

Esperamos que este artigo possa servir de apoio aos interessados em compreender de forma qualitativa e quantitativa as característica do modelo de Ptolomeu, assim como, algumas características da revolução copernicana do século XVI. Também esperamos que o material possa estimular discussões em diferentes níveis e em diversas disciplinas associadas com a evolução do pensamento científico.

\footnotetext{
${ }^{11}$ Curiosamente, Apolônio de Perga foi quem impulsionou a teoria dos epiciclos e excêntricos e também foi o responsável pelo estudo detalhado das cônicas na antiga Grécia, tendo influenciado posteriormente os estudos de Kepler.
} 


\section{Agradecimentos}

A.M.V.T. Gostaria de agradecer à UFES por permitir coordenar um projeto de extensão da Pró-reitoria de extensão sobre história da ciência. Este trabalho foi iniciado dentro deste programa.

\section{Referências}

[1] Ptolemy, em: Great Books of the Western World (William Benton, Chicago, 1952) v. 16, p. 497.

[2] T.L. Heath, Aristarchus of Samos: the ancient Copernicus (Courier Corporation, Massachusetts, 2004).

[3] A.M. Velásquez-Toribio e M.V. Oliveira, Rev Bras Ens Fís 41, e20180096 (2019).

[4] O. Neugebauer, Astronomy and History Selected Essays (Springer, Lansing, 1983).

[5] C. Linton, From Eudoxus to Einstein: A History of Mathematical Astronomy (Cambridge University Press, Cambridge, 2004).

[6] S. Weinberg, Para explicar o mundo: a descoberta da ciência moderna(Companhia das Letras, São Paulo, 2015).

[7] O. Gingerich, Copernicus: A Very Short Introduction (Oxford University Press, Oxford, 2016).

[8] J. Al-Khalili, The House of Wisdom (Penguin Publishing Group, Nova York, 2011).

[9] R. Rashed, Encyclopedia of the History of Arabic Science (Routledge, Londres, 1996).

[10] N.M. Swerdlow, Journal for the History of Astronomy 48, 33 (2017).

[11] N. Copernicus, As revoluçôes dos orbes celestes (Fundação Calouste Gulbenkian, Lisboa, 1996).

[12] N.M. Swerdlow, em: Wrong for the Right Reasons (Archimedes) editado por J.Z. Buchwald e A. Franklin (Springer, Dordrecht, 2005), v. 11.

[13] J.L.M. Dreyer, A history of astronomy from Thales to Kepler (Courier Corporation, Londres, 1953), v. 2, p. 465.

[14] T. Khun, A estrutura das Revoluções científicas (Editora Perspectiva S.A, São Paulo, 2019).

[15] O. Gingerich, Vistas in Astronomy 17, 85 (1975).

[16] E. Poulle e U. Gingerich, Comptes rendus des séances 48, 531 (1967)

[17] N.R. Hanson, The Mathematical Power of Epicyclical Astronomy 51, 150 (1960).

[18] M.M. Woolfson e M.S. Woolfson, Mathematics for Physics (Oxford University Press, Oxford, 2007). 